\title{
A calibration method for broad-bandwidth cavity enhanced absorption spectroscopy performed with supercontinuum radiation
}

\author{
T. Laurila • I.S. Burns · J. Hult • J.H. Miller • \\ C.F. Kaminski
}

Received: 4 December 2009 / Revised version: 16 April 2010 / Published online: 22 May 2010

(c) Springer-Verlag 2010

\begin{abstract}
An efficient calibration method has been developed for broad-bandwidth cavity enhanced absorption spectroscopy. The calibration is performed using phase shift cavity ring-down spectroscopy, which is conveniently implemented through use of an acousto-optic tunable filter (AOTF). The AOTF permits a narrowband portion of the SC spectrum to be scanned over the full high-reflectivity bandwidth of the cavity mirrors. After calibration the AOTF is switched off and broad-bandwidth CEAS can be performed with the same light source without any loss of alignment to the set-up. We demonstrate the merits of the method by probing transitions of oxygen molecules $\mathrm{O}_{2}$ and collisional pairs of oxygen molecules $\left(\mathrm{O}_{2}\right)_{2}$ in the visible spectral range.
\end{abstract}

\author{
T. Laurila ( $\varangle) \cdot$ J. Hult · C.F. Kaminski \\ Department of Chemical Engineering and Biotechnology, \\ University of Cambridge, Pembroke Street, CB2 3RA Cambridge, \\ UK \\ e-mail: tk125@cam.ac.uk \\ Fax: +44-1223-334796 \\ C.F. Kaminski \\ e-mail: cfk23@cam.ac.uk
}

\section{I.S. Burns}

Department of Chemical and Process Engineering, University of Strathclyde, Montrose Street, G1 1XJ Glasgow, Scotland

\section{J.H. Miller}

Department of Chemistry, George Washington University,

Washington, 20052 DC, USA

C.F. Kaminski

SAOT School of Advanced Optical Technologies, Max Planck Institute for the Science of Light,

Guenther-Scharowsky-Strasse 1, 91058 Erlangen, Germany

\section{Introduction}

Direct absorption measurement is a simple method to quantify species concentrations on an absolute scale. The use of optical resonators makes it possible to increase the effective absorption path length by orders of magnitude, raising the sensitivity of the technique to levels where trace gas concentrations can be measured. Cavity ring-down spectroscopy (CRDS) [1] is a popular variant of this methodology and is today a well-established method for concentration measurements of trace species [2-5]. In CRDS the intensity decay of a light pulse transmitted through the cavity is measured in the presence and absence of absorbers. If absorbers are present, the cavity lifetime, i.e., the photon residence time in the cavity, shortens and the species concentration can be determined assuming the absorption cross sections of the probed transitions are known.

A variant of CRDS is phase shift cavity ring-down spectroscopy (PS-CRDS), which involves the amplitude modulation of a continuous-wave (cw) light source and measurement of the phase delay of the transmitted intensity through the cavity [6, 7]. In most CRDS implementations spectral transitions are scanned sequentially to avoid the occurrence of complex multi-exponential intensity decays [8-10]. The need for sequential scanning and the associated loss in time resolution are disadvantageous, but this is offset by the fact that CRDS measurements are self calibrating, as one simply compares the ring-down times of empty and absorber containing cavities. Emerging broadband variants of CRDS offer exciting possibilities to record multi-species absorption spectra $[1,11]$.

Another way of performing absorption measurements in optical cavities is cavity enhanced absorption spectroscopy (CEAS). CEAS is a technique related to CRDS, but here intensity changes are measured rather than ring-down times 
$[12,13]$. This makes CEAS simple to implement. A further advantage is the possibility to perform multiplexed measurements by simply injecting broad-bandwidth light into the cavity. Incoherent sources such as light-emitting diodes (LEDs) [14-16] or filament lamps [17] have been used for broadband CEAS. Light transmitted through the cavity is subsequently dispersed and recorded on an array detector, and thus, full spectra can be recovered at once without any need for sequential scanning. Compared to CRDS, disadvantages of CEAS are that it requires a calibration measurement and is sensitive to intensity noise of the light source. The calibration is performed either by using an absorber at known concentration or through a measurement of the cavity mirror reflectivity.

In this paper we focus on the use of supercontinuum radiation generated in photonic crystal fibres [18] for multiplexed broad-bandwidth absorption measurements using CEAS. Supercontinuum sources offer significant advantages in optical sensing over conventional, narrow linewidth sources, such as diode lasers [19, 20], in enabling measurements at a very high measurement speed or multiplexed measurements [21-24]. Only a few demonstrations have been reported so far on the use of broadband light sources such as stabilised frequency combs or supercontinuum light in CEAS or CRDS. Gherman and Romanini demonstrated the locking of a frequency comb to a high-finesse optical cavity for CEAS [25]. Thorpe et al. used an optical frequency comb and a streak camera to perform broadband CRDS [26]. In their work a very high (>50\%) optical throughput was obtained by matching the frequency comb to the longitudinal mode structure of the cavity. However, active locking and stabilisation of the frequency components make these techniques complex to implement in practical sensing environments. More recently, supercontinuum CRDS has been realised without active matching of the longitudinal modes of the light source and the cavity [27-29]. Compared to the mode matched approach, the trade-off is a decreased optical throughput. Johnston and Lehmann used a supercontinuum source and CRDS to calibrate a novel prism cavity for broad-bandwidth CEAS [27]. In that work a spectrometer and photon counting system were used to determine the ring-down times as a function of wavelength at $1 \mathrm{~nm}$ resolution. Stelmaszczyk et al. demonstrated broadband CRDS with supercontinuum radiation generated by filaments $[28,29]$. They used an ICCD camera and a spectrometer featuring a resolution of $0.01-0.25 \mathrm{~nm}$ for the detection of $\mathrm{NO}_{2}$ [29].

We have recently exploited supercontinuum radiation sources in broadband CEAS (SC-CEAS) of gases [23, 24] and liquids [30,31]. In the gas phase, SC-CEAS can be used for rapid acquisition of spectra over bandwidths covering approximately $100 \mathrm{~nm}$. SC-CEAS allows multiple species to be detected at trace concentrations over time scales of a second or even less [23]. The bandwidth of SC-CEAS is limited by the reflectivity range of available high-quality cavity mirrors, which typically have a bandwidth of about $10 \%$ of the central wavelength. The bandwidth limitation can be overcome by exploiting total internal reflection in prism cavities [27, 32].

In the past we have accomplished calibration of SCCEAS using known concentrations of broadband absorbers [23] but this is restricted by the non-availability of suitable calibration species in some spectral regions of interest. A different approach is to perform a separate CRDS or PSCRDS measurement while maintaining the alignment of the set-up $[14,15]$. In this paper we present a novel approach to calibrate SC-CEAS using the same broadband light source. A supercontinuum radiation source and an acousto-optic tunable filter (AOTF) are used to perform PS-CRDS. The AOTF isolates a narrow bandwidth from the supercontinuum radiation and scans the centre frequency across the required measurement bandwidth whilst modulating the transmitted intensity. After calibration with narrow bandwidth CRDS, the system is switched over into a broad-bandwidth CEAS configuration, without any loss in optical alignment.

\section{Background theory}

Phase shift cavity ring-down spectroscopy involves the periodic intensity modulation of the light coupled into an optical cavity. It can be shown (see e.g. $[1,33]$ ) that the intensity of transmitted light is described by the convolution of an exponential decay function of the cavity and the modulation waveform. The resulting phase shift $\theta$ induced by the optical cavity is given by:

$\tan \theta(\lambda)=-\omega \tau(\lambda)$

where $\omega=2 \pi f$ is the angular modulation frequency and $\tau$ the photon residence time (lifetime) in the optical cavity. It is worth noting that the same equation is used in other frequency-domain lifetime measurement techniques, such as fluorescence microscopy [33]. Just as in other configurations of CRDS, the lifetime is given by [1]:

$\tau(\lambda)=\frac{d}{c\left[\left|\ln \left(R_{\mathrm{eff}}(\lambda)\right)\right|+\sum_{i} \sigma_{i}(\lambda) \int_{0}^{d} n_{i}(x) d x\right]}$,

where $c$ is the speed of light, $d$ the cavity length, $R_{\text {eff }}$ the effective cavity reflectivity, $\sigma_{i}$ the absorption cross section, and $n_{i}$ the number density of an absorbing species $i$. The effective reflectivity $R_{\text {eff }}$ depends not only on the geometric mean of the mirror reflectivities but also on the cavity alignment and the coupling of the laser beam to the cavity. In addition to absorption, Rayleigh scattering from the gas molecules can affect the observed decay times, especially 
at shorter wavelengths due to the $\lambda^{-4}$ wavelength dependence. Since the effective reflectivity is typically close to unity, the reflectivity term in the denominator of (2) may be approximated by $\left(1-R_{\text {eff }}\right)$. For cases where only one homogeneously absorbing species is present, the expression can thus be simplified to:

$\tau(\lambda)=\frac{d}{c\left[\left(1-R_{\mathrm{eff}}(\lambda)\right)+\alpha(\lambda) d\right]}$,

where $\alpha$ is the absorption coefficient. By combining equations (1) and (3) we find that the absorption coefficient resulting from PS-CRDS can be expressed as:

$\alpha_{\text {PS-CRDS }}(\lambda)=\frac{\omega}{c}\left[\frac{1}{\tan \theta_{\text {sample }}(\lambda)}-\frac{1}{\tan \theta_{\text {empty }}(\lambda)}\right]$,

where $\theta_{\text {sample }}$ is the phase shift with the absorber present in the cavity and $\theta_{\text {empty }}$ the phase shift of an empty cavity. The sensitivity of the phase shift to changes in cavity lifetime can be defined by differentiating equation (1):

$\frac{d \theta}{d \tau}=\frac{\sin \theta \cos \theta}{\tau}$.

It is thus clear that the sensitivity will be optimised for $\theta=$ $\pi / 4$. Therefore, an angular modulation frequency $\omega$ should be chosen to result in $\omega \tau \approx 1$ [7].

In the alternative case of cavity enhanced absorption spectroscopy (CEAS), where the transmitted intensity is recorded rather than the cavity decay time, the absorption coefficient at the low-loss and high-reflectivity limit [17] may be calculated according to the following equation:

$\alpha_{\mathrm{CEAS}}(\lambda)=\left[\frac{I_{0}(\lambda)}{I(\lambda)}-1\right] \frac{1-R_{\mathrm{eff}}(\lambda)}{d}$,

where $I_{0}$ is the intensity transmitted through an empty cavity and $I$ is the intensity transmitted with the absorber present. Unlike cavity ring-down measurements, CEAS is not a self calibrating technique and the effective cavity reflectivity $R_{\text {eff }}$ must be determined by another measurement. Here we show that supercontinuum PS-CRDS can be used to determine the effective reflectivity, and thus, enable calibrated broadband CEAS.

\section{Experimental set-up}

The experimental set-up for SC-CEAS using PS-CRDS calibration is shown in Fig. 1. The fibre laser based SC light source (Fianium SC400) has a $10 \mathrm{MHz}$ repetition rate and 2 Watt total output power between 400 and $2500 \mathrm{~nm}$. The fibre collimator (Fianium) produces a collimated output beam having a diameter of around $2 \mathrm{~mm}$ in the visible spectral region. For the experiments presented here a short-pass mirror is used to remove wavelengths longer than $800 \mathrm{~nm}$.
The beam is guided through an acousto-optic tunable filter (AOTF; AA Opto Electronic nc-VIS-TN, 450-710 nm operating range). For the reflectivity calibration the set-up is run in the PS-CRDS mode where the spectrally narrow 1 st order beam transmitted through the AOTF is selected (see beam path B in Fig. 1). The RF driver of the AOTF has eight channels each of which can be independently controlled. In this work only a single AOTF channel having $1.6 \mathrm{~nm}$ FWHM spectral bandwidth was used. Spectrally multiplexed operation could be achieved by using multiple channels simultaneously. The centre wavelength of the 1st order beam can be electronically adjusted by changing the frequency of the RF signal driving the AOTF. The AOTF also allows for intensity modulation of the 1 st order beam for which a signal generator with a sinusoidal output waveform is used. After the AOTF the spectrally narrow beam is coupled into a single-mode optical fibre, the output of which is coupled to the cavity using a focusing lens and two beam steering mirrors. A $1.13 \mathrm{~m}$ long optical cavity is formed by two plano-concave mirrors (Layertech, $1 \mathrm{~m}$ radius of curvature, 1 inch diameter, $>99.97 \%$ nominal reflectivity from 620 to $680 \mathrm{~nm}$ ). In the PS-CRDS mode of operation, light transmitted through the cavity is coupled into a multi-mode fibre and detected using a photomultiplier tube.

The phase shift of the transmitted signal with respect to the modulated waveform at the cavity input is measured with a lock-in amplifier (Stanford Research Systems SR830). Here the reference or zero phase angle $\theta_{0}$ was acquired after removing one of the cavity mirrors. The reference phase angle could be monitored without the mirror removal by placing a beam splitter between the AOTF and the cavity to reflect part of the laser beam onto a reference detector. The lock-in amplifier and the RF driver of the AOTF are connected to a personal computer and the whole experiment is controlled with a LabVIEW program. The program causes the AOTF to step through a series of different wavelengths and for each wavelength the phase angle is read from the lock-in amplifier. Here, a lock-in amplifier time constant of $3 \mathrm{~s}$ is used and $15 \mathrm{~s}$ settling time is allowed between each wavelength step. A modulation frequency of $6-25 \mathrm{kHz}$, depending on the quality of the cavity alignment, is used to generate a phase shift of around $45^{\circ}$, thus maximising the sensitivity as described above. The modulation frequency of the AOTF is slow compared to the repetition rate of the pulsed supercontinuum source, and therefore, the excitation can be considered as continuous.

In the CEAS mode of operation the spectrally broader 0th order beam of the AOTF is coupled into the single-mode fibre (see beam path A in Fig. 1). A broadband interference filter ( $40 \mathrm{~nm}$ FWHM) is used to match the bandwidth of the 0th order beam with the high-reflectivity range of the mirrors. The use of the single-mode fibre to couple both the 1st (PS-CRDS mode) and 0th order beam (CEAS mode) into 


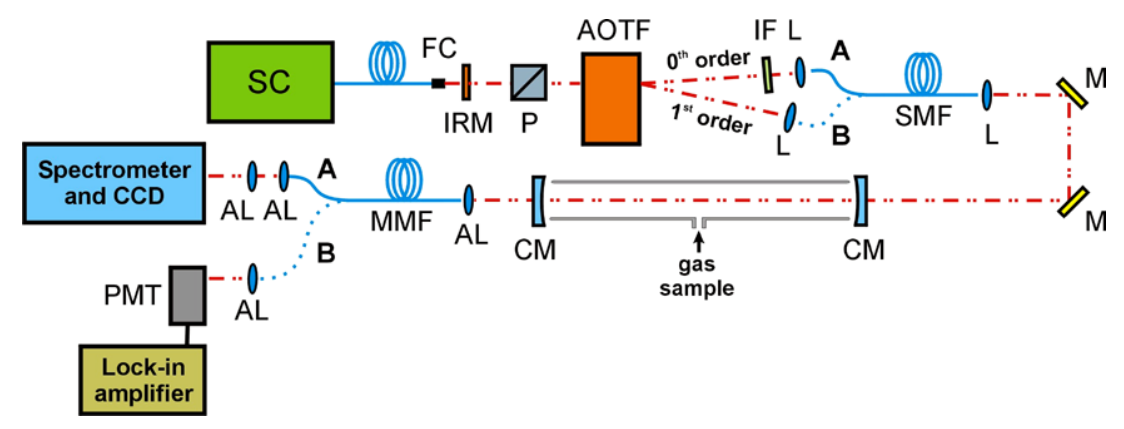

Fig. 1 Experimental set-up for broadband CEAS using PS-CRDS calibration. Beam path $\mathrm{B}$ is for the calibration mode where the modulated 1st order beam is coupled into the cavity and the phase shift is recorded using a PMT and lock-in amplifier. In the CEAS mode beam path A is used where the spectrally broader, 0th order beam is coupled into the cavity. The spectrum of transmitted light is recorded with a spectrometer and a cooled CCD camera. SC, supercontinuum source

the cavity ensures that the launching condition remains the same during the reflectivity calibration and the subsequent broadband CEAS measurement. This is important as the effective reflectivity $R_{\text {eff in (6) depends both on the alignment }}$ of the cavity and the coupling to the cavity. For CEAS, light transmitted through the cavity is coupled into a $62.5 \mu \mathrm{m}$ multi-mode fibre and the spectrum is acquired using a grating spectrometer fitted with a cooled CCD camera.

\section{Results and discussion}

The reflectivity of the cavity was first measured using PSCRDS. The acquired phase shifts and corresponding reflectivity values ((1) and (2)) for a nitrogen-flushed cavity are plotted against wavelength in Fig. 2. The reflectivity peaks at 0.999860 around $655 \mathrm{~nm}$, and is above 0.9997 over a $70 \mathrm{~nm}$ wide region, which agrees very well with the manufacturer's specifications. The uncertainty in the reflectivity values was estimated by calculating the standard deviation of 512 consecutive phase angle values read at $2 \mathrm{~Hz}$ rate from the buffer of the lock-in amplifier. The error bars $( \pm 3 \sigma)$ in Fig. 2 correspond to the observed standard deviation at each wavelength. At peak reflectivity the standard deviation $(3 \sigma)$ corresponds to a 0.6 degree uncertainty in the phase and $3 \times 10^{-6}$ uncertainty in the reflectivity, respectively. The uncertainty is dominated by the precision of the phase angle measurement with smaller uncertainties related to the cavity length and the modulation frequency.

The spectral resolution of PS-CRDS using a single channel of the AOTF is limited to $1.6 \mathrm{~nm}$ FWHM. However, after the reflectivity calibration the set-up can be switched into the SC-CEAS mode and spectra can be acquired at higher resolution. Figure 3 shows the magnetic dipole transition $b^{1} \Sigma_{g}^{+}\left(v^{\prime}=1\right)-X^{3} \Sigma_{g}^{-}\left(v^{\prime \prime}=0\right)$ of $\mathrm{O}_{2}$ around $690 \mathrm{~nm}$
(Fianium SC 400); FC, fibre collimator (Fianium); IRM, infrared mirror (Comar 716 GK 25); P, cube polariser; AOTF, acousto-optic tunable filter; IF, band pass interference filter (Comar 633 IU 12); L, lens; SMF, single-mode fibre (Thorlabs PI-630A-FC-10); M, mirror; CM, cavity mirror; AL, achromatic lens; MMF, multi-mode fibre (Thorlabs M31L05); PMT, photomultiplier tube (Hamamatsu R636-10 with C7319 preamplifier)

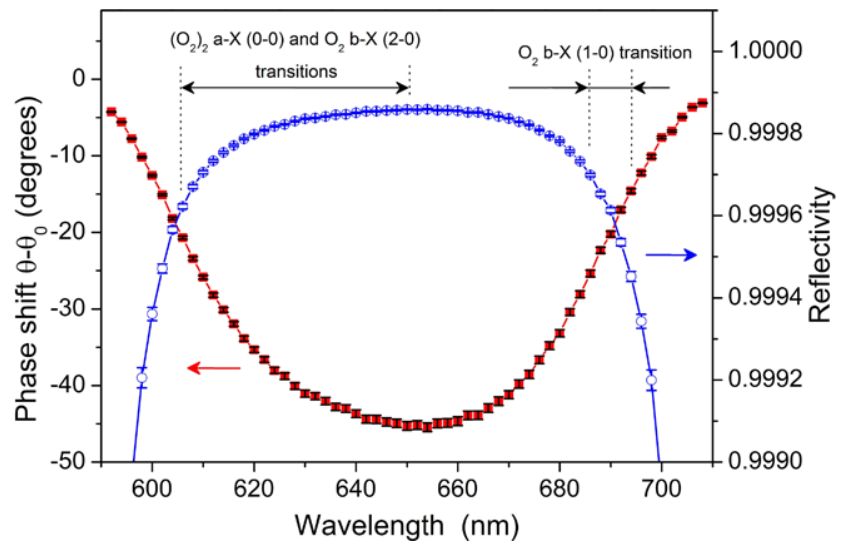

Fig. 2 Typical phase shift of a cavity flushed with a 3 standard litres per minute (SLPM) flow of nitrogen. The phase shifts (red squares) were recorded over the high-reflectivity range of the mirrors at $2 \mathrm{~nm}$ steps. The corresponding effective cavity reflectivity is shown by the blue circles. The modulation frequency $f$ was $6 \mathrm{kHz}$. In a $1.13 \mathrm{~m}$ long cavity the peak reflectivity of $99.986 \%$ around $655 \mathrm{~nm}$ yields a cavity lifetime of $26.9 \mu$ s corresponding to an effective absorption path length of $8.1 \mathrm{~km}$. The spectral regions of the investigated transitions of $\left(\mathrm{O}_{2}\right)_{2}$ and $\mathrm{O}_{2}$ are also indicated

recorded using SC-CEAS after the PS-CRDS reflectivity calibration. The individual absorption lines were resolved at ambient conditions by using a $1.25 \mathrm{~m}$ focal length grating spectrometer (SPEX 1269) with a narrow input slit setting, which resulted in a $0.007 \mathrm{~nm}(4.4 \mathrm{GHz})$ FWHM experimental resolution. Even at this high resolution the spectral brightness of the SC source allowed for $1 \mathrm{~s}$ exposure times on the CCD camera (Andor iXon DV885, $1004 \times 1002$ pixels). For the results presented here no electron multiplying gain of the Andor CCD cameras was used.

The high resolution limited the spectral coverage of the detection set-up to about $1 \mathrm{~nm}$, and therefore, the spectrum shown in Fig. 3 was acquired in 6 separate sections. The experimental spectrum was compared to a spectrum cal- 

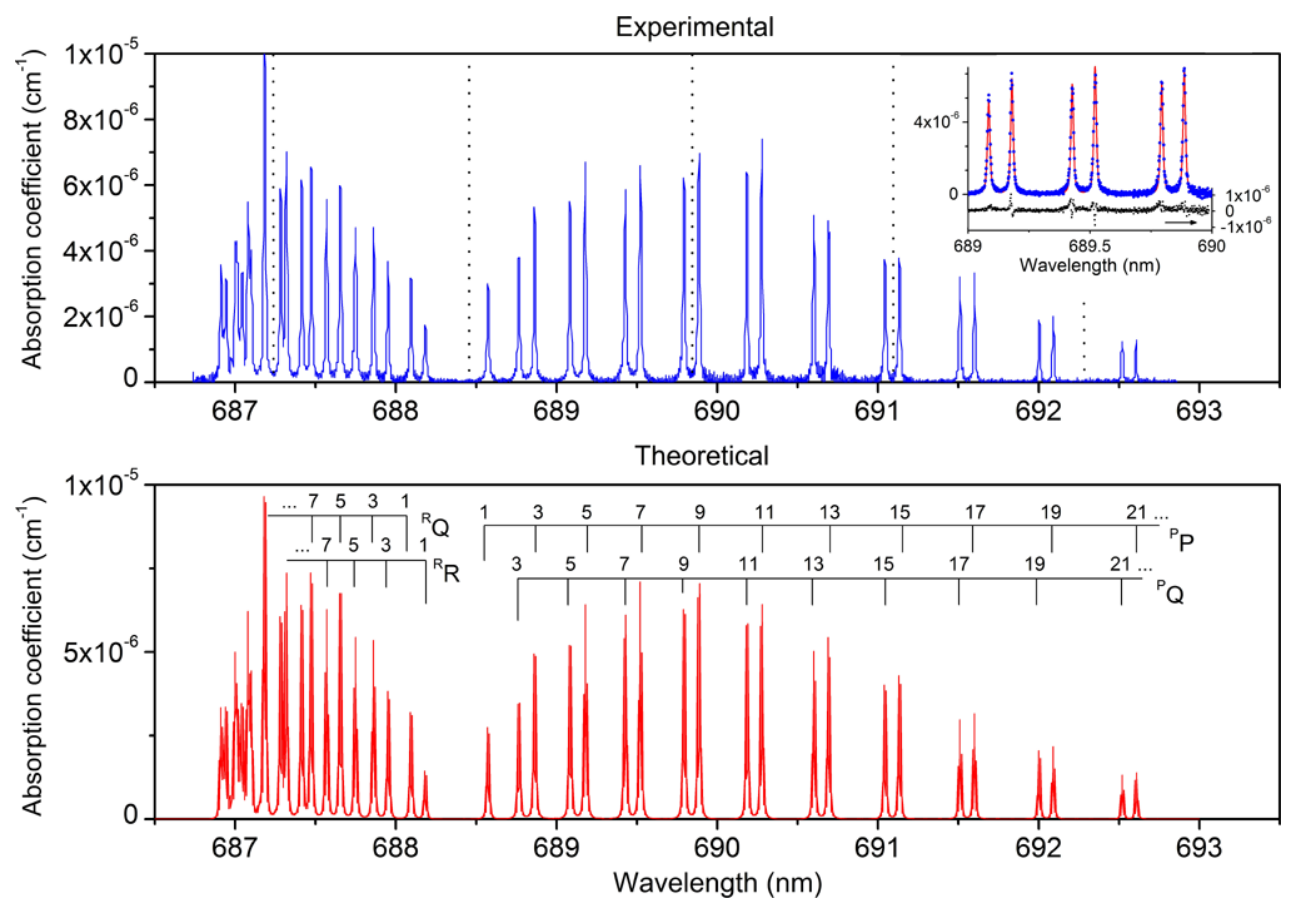

Fig. 3 Upper panel: Experimental CEAS spectrum of the $b-X$ (1-0) transition of $\mathrm{O}_{2}$ around $690 \mathrm{~nm}\left(14534.9 \mathrm{~cm}^{-1}\right)$. The cavity was flushed with a 3 SLPM flow of $100 \%$ oxygen at 1 atm. The full spectrum was acquired in 6 separate sections, as indicated by the vertical dotted lines. For each spectral section the exposure time of the CCD camera was $1 \mathrm{~s}$. Wavelength dependent etalon effects, which are thought to be due to the multi-mode fibre and CCD chip, were observed causing some variation in the background noise levels between the spectral sections. The baseline of the spectrum where no absorp-

culated using spectral parameters from the HITRAN 2004 database [34]. As the spectral width of the detection was comparable to the width of the $\mathrm{O}_{2}$ absorption lines the theoretical spectrum was corrected for non Beer-Lambert behaviour [11] using the following methodology. First, the theoretical transmission spectrum was calculated from the single pass absorbance spectrum, predicted using HITRAN, and the mirror reflectivity curve. The shape of the $\mathrm{O}_{2}$ absorption lines was modeled by a Voigt profile. This full resolution transmission spectrum was then convoluted with the spectrometer lineshape function to account for the finite detector resolution [24]. Finally the theoretical absorption coefficient was calculated from this convoluted transmission spectrum using (6), and is plotted in the lower panel of Fig. 3. A good agreement between the experimental and theoretical spectra is observed.

Despite the modest spectral resolution in the PS-CRDS mode of operation the set-up can be used for PS-CRDS of broadband absorbers. Applications in the analysis of liquids $[30,31]$ can be envisaged as sub-nm resolution is not typically required in the liquid phase. Here AOTF based PS-CRDS was demonstrated in the gas phase by probing the broadband absorption of the collisional pair of oxygen, tion features were observed $(688.3-688.5 \mathrm{~nm})$ has a standard deviation of $5 \times 10^{-8} \mathrm{~cm}^{-1}$. Lower panel: A theoretical spectrum calculated using spectral parameters from the HITRAN 2004 database [34]. Fitting the theoretical and experimental spectra yields an experimental resolution of $0.007 \mathrm{~nm}$ FHWM. Some components of the ${ }^{\mathrm{P}} \mathrm{P},{ }^{\mathrm{P}} \mathrm{Q},{ }^{\mathrm{R}} \mathrm{R}$, and ${ }^{\mathrm{R}} \mathrm{Q}$ branches are indicated together with their $N^{\prime \prime}$ quantum numbers $[34,35]$. The inset in the upper panel shows a magnified view of the 689-690 nm section of the experimental (blue dots) and theoretical (red line) spectra as well as the difference of the two

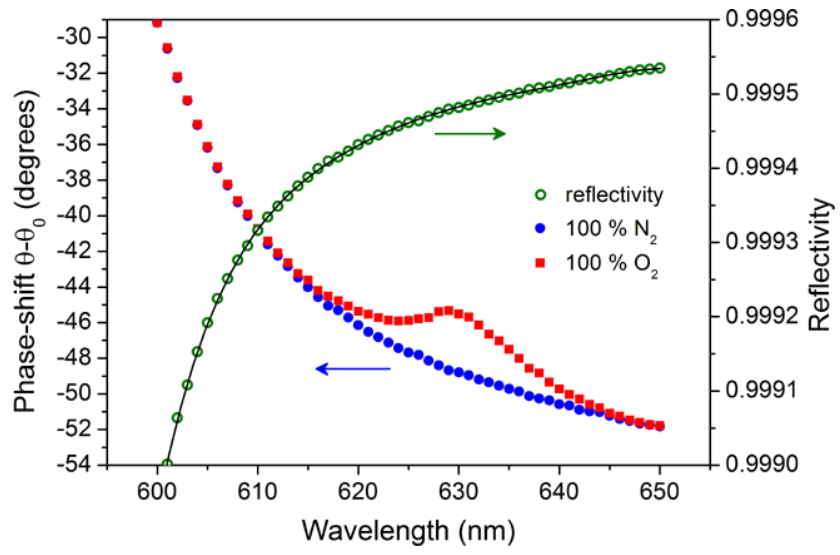

Fig. 4 Phase shift of a nitrogen (blue dots) and oxygen (red squares) filled cavity acquired at $1 \mathrm{~nm}$ steps. The effective cavity reflectivity (green circles) corresponding to the nitrogen-flushed cavity is shown together with a polynomial fit (solid line). Here the cavity alignment was not as good as in Fig. 2, and therefore, a slightly higher modulation frequency of $25 \mathrm{kHz}$ was used. The ambient temperature and pressure were $21.2^{\circ} \mathrm{C}$ and 1017 mbar, respectively

$\left(\mathrm{O}_{2}\right)_{2}$. The phase shifts of the cavity flushed with $100 \%$ nitrogen and 100\% oxygen, respectively, are shown in Fig. 4. 


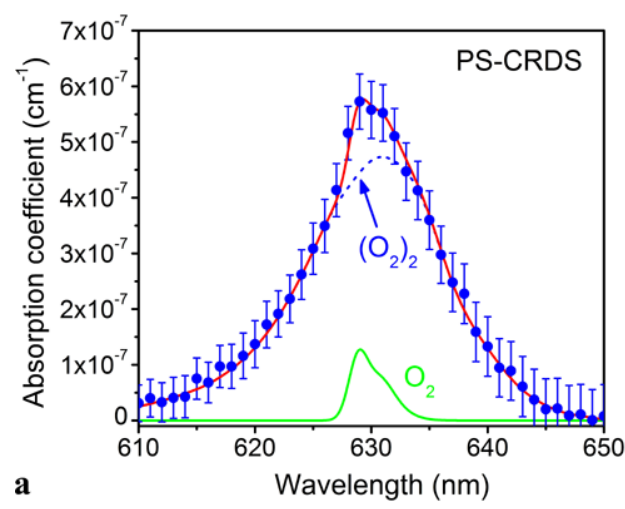

Fig. 5 (a) PS-CRDS spectrum of 100\% oxygen around $630 \mathrm{~nm}$ $\left(15873 \mathrm{~cm}^{-1}\right)$ corresponding to the phase shift data shown in Fig. 4. The instrument broadened $b-X(2-0)$ band of $\mathrm{O}_{2}$ is shown by the green line at the bottom. The absorption profile of the $a-X(0-0)$ transition of $\left(\mathrm{O}_{2}\right)_{2}$ is shown by the dotted blue line [36]. The sum of the two transitions is shown by the red line, which agrees well with the experimental data (blue dots). The error bars correspond to the standard deviation $( \pm 1 \sigma)$ of the 512 phase shift values read from the buffer of the lockin amplifier. (b) Supercontinuum CEAS spectrum of oxygen acquired

The effective cavity reflectivity corresponding to the phase shift of the nitrogen-flushed empty cavity is also shown. The reflectivity curves in Figs. 2 and 4 are not corrected by the small Rayleigh scattering contribution due to nitrogen molecules. The resulting PS-CRDS spectrum, calculated using (4), is shown in Fig. 5a. The observed absorption spectrum actually consists of two transitions, the broad $a^{1} \Delta_{g}+a^{1} \Delta_{g}\left(v^{\prime}=0\right)-X^{3} \Sigma_{g}^{+}+X^{3} \Sigma_{g}^{+}\left(v^{\prime \prime}=0\right)$ transition of the collisional pair of oxygen molecules $\left(\mathrm{O}_{2}\right)_{2}$ and the $b^{1} \Sigma_{g}^{+}\left(v^{\prime}=2\right)-X^{3} \Sigma_{g}^{-}\left(v^{\prime \prime}=0\right)$ band of $\mathrm{O}_{2}$. However, the contribution of the latter is fairly small because the instrumental resolution (1.6 nm FWHM) is much larger than the width of the absorption lines of this band. The $\mathrm{O}_{2} b-X$ (2-0) band convoluted with the instrument function is shown at the bottom of Fig. 5a. The dashed line shows the absorption profile of the collisional pair, which has been measured in a previous study using CRDS [36]. The sum of these two transitions (red line) agrees well with the observed absorption coefficient of $5.6 \times 10^{-7} \mathrm{~cm}^{-1}$ at $630 \mathrm{~nm}$. The Rayleigh scattering cross section of nitrogen is about $10 \%$ larger than that of oxygen [36], which produces a negative offset in the oxygen spectra of Fig. 5. However, this contribution (about $1 \times 10^{-8} \mathrm{~cm}^{-1}$ ) is so small that it was not taken into account here. The error bars indicate the uncertainty in the absorption coefficient resulting from the uncertainty in the phase angle measurement.

Figure $5 \mathrm{~b}$ shows the SC-CEAS spectrum of pure oxygen at $630 \mathrm{~nm}$. For this measurement the cavity reflectivity was again determined using PS-CRDS as described above. The CEAS spectrum was recorded with a grating spectrometer

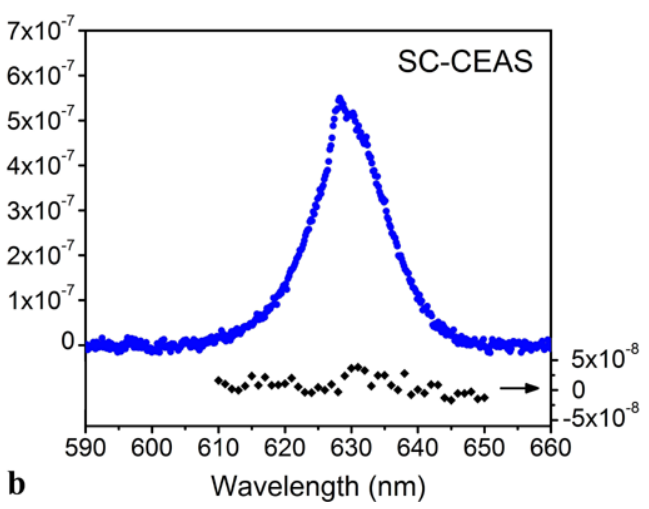

using $1 \mathrm{~s}$ exposure time. The difference between the PS-CRDS and SCCEAS spectra is also shown. The difference spectrum was corrected by the small offset due to the different Rayleigh scattering cross sections of nitrogen and oxygen. The experimental conditions in the SC-CEAS measurement were: spectrometer resolution $2 \mathrm{~nm}$ FWHM, 100\% oxygen flow at $3 \mathrm{SLPM}, 20.4^{\circ} \mathrm{C}$ ambient temperature and 1003 mbar pressure. The standard deviation of the region where no absorption features are found $(590-605 \mathrm{~nm})$ is $7 \times 10^{-9} \mathrm{~cm}^{-1}$

(Andor Shamrock 303i) and a cooled CCD camera (Andor iXon DV887, $512 \times 512$ pixels). The $70 \mathrm{~nm}$ wide SC-CEAS spectrum was recorded in $1 \mathrm{~s}$. There is a good agreement between the CEAS and PS-CRDS spectra of $\left(\mathrm{O}_{2}\right)_{2}$. In Fig. 5b the standard deviation of the baseline is $7 \times 10^{-9} \mathrm{~cm}^{-1}$, which is better than for the higher resolution spectrum in Fig. 3. This is expected because at the lower resolution of Fig. $5 \mathrm{~b}$ a larger number of photons reach the detector in each spectral bin.

Both CRDS and PS-CRDS have their advantages and disadvantages. CRDS is very sensitive technique owing to the precise determination of ring-down times and the immunity against the intensity noise of the source. The main factors determining the precision of PS-CRDS include the uncertainties of the phase shift values, modulation frequency and cavity length. The performance of PS-CRDS is typically limited by the precision of the phase angle measurement [2]. Using a blue LED for $\mathrm{NO}_{2}$ sensing it has been shown, however, that PS-CRDS can achieve very good sensitivity [37]. On the other hand, CRDS can give visual information on the quality of the cavity alignment, that is, whether the decay is single or multi-exponential or if mode-beating effects take place. This information can be useful during the alignment of the optical cavity. A single-phase angle reading in PSCRDS does not give such information. However, some additional information on the quality of the cavity alignment and intensity decay of a PS-CRDS set-up can be obtained by studying the phase shift as a function of the modulation frequency [38]. Both this approach and the measurement of the demodulation are well known methods in fluorescence 


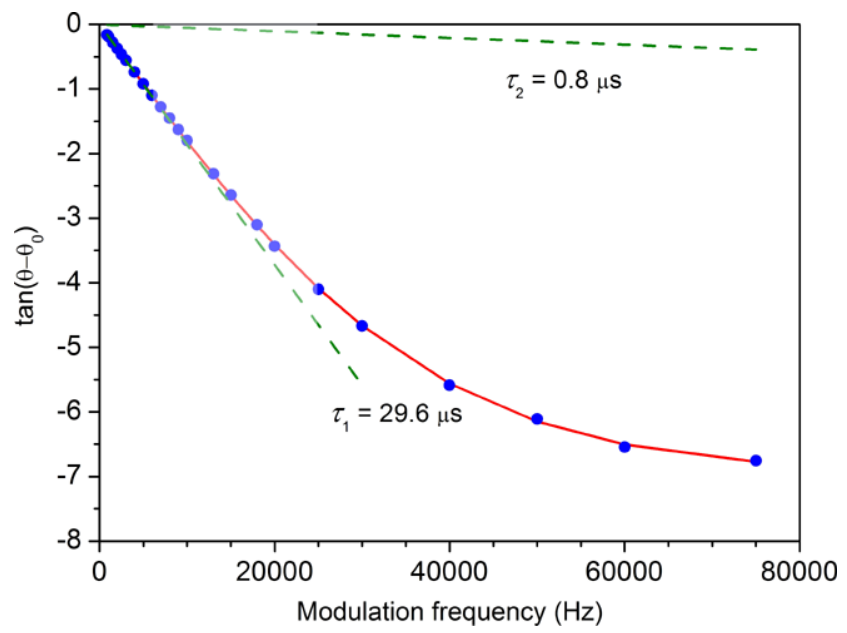

Fig. 6 Phase shift of a nitrogen-flushed cavity measured at $630 \mathrm{~nm}$ as a function of the modulation frequency $f$. The red line shows a bi-exponential fit $(N=2$ in (7)) to the experimental data (dots). Dashed lines show the expected phase shifts due to single-exponential decays corresponding to $\tau_{1}$ and $\tau_{2}$, respectively. The shaded area shows the modulation frequency range used in this work

lifetime measurements [33]. In the case of multi-exponential decay the phase angle can be expressed as:

$\tan \theta(\omega)=\frac{-\sum_{i}^{N} \frac{\omega \beta_{i} \tau_{i}^{2}}{\omega^{2} \tau_{i}^{2}+1}}{\sum_{i}^{N} \frac{\beta_{i} \tau_{i}}{\omega^{2} \tau_{i}^{2}+1}}$,

where $\beta_{i}$ is the initial amplitude of the single-exponential decay component $i$. The fractional steady-state intensity of each decay component $i$ can be defined as [38]:

$\gamma_{i}=\frac{\beta_{i} \tau_{i}}{\sum_{i}^{N} \beta_{i} \tau_{i}}$

Figure 6 shows the tangent of the phase shift as a function of the modulation frequency. The modulation frequency $f$ was limited to below $100 \mathrm{kHz}$ by the frequency response of the PMT preamplifier and the lock-in amplifier. It can be seen that the response is well described by a bi-exponential fit to the data. The lifetimes and steady-state fractions resulting from the fit are $\tau_{1}=29.6 \mu \mathrm{s}, \tau_{2}=0.8 \mu \mathrm{s}, \gamma_{1}=99.3 \%$, $\gamma_{2}=0.7 \%$, respectively. The first lifetime $\tau_{1}$ corresponds to the photon residence time in the optical cavity. The second apparent lifetime is caused by the limited frequency response of the PMT preamplifier. The $-3 \mathrm{~dB}$ cut-off frequency corresponding to $\tau_{2}=0.8 \mu$ s is $199 \mathrm{kHz}$, which agrees well with the nominal $200 \mathrm{kHz}-3 \mathrm{~dB}$ frequency of the PMT preamplifier (Hamamatsu, C7319).

In this paper we have used a non-confocal cavity geometry, which results in a densely spaced, quasi-continuous cavity mode structure [39], and thus, even the 1st order AOTF beam excites a large number of longitudinal cavity modes. The supercontinuum beam was focused to the centre of the cavity to prevent excessive excitation of higher order transverse modes. Generally speaking, the excitation of multiple cavity modes can cause mode beating and non-exponential intensity decays [40-42]. However, in a non-stabilised cavity, like the one used here, mode-beating effects tend to average out [40]. Recently, we have studied the direct recording of ring-down times of $12 \mathrm{~nm}$ wide spectrally filtered subns supercontinuum pulses in a similar passive non-confocal cavity [24]. In that work, the intensity decays were recorded at $1550 \mathrm{~nm}$ using a fast $8 \mathrm{GHz}$ photodiode and real-time oscilloscope and were found to be essentially single exponential for the non-confocal cavity employed.

\section{Conclusions}

A method has been developed for calibrating the effective absorption length in broadband cavity enhanced absorption spectroscopy using supercontinuum radiation. Both the reflectivity calibration of the cavity and the cavity enhanced absorption measurement can be accomplished over a wide spectral range using the same light source whilst maintaining the same light coupling to the cavity. The resolution of the PS-CRDS was $1.6 \mathrm{~nm}$, more than sufficient to calibrate the smoothly varying cavity mirror reflectivity. Commercially available supercontinuum sources and acoustooptic filters permit extension of the PS-CRDS calibration to cover spectral regions from the near-ultraviolet to the nearinfrared. The latter is particularly promising as many molecules of analytical interest have their vibrational overtone transitions in the near-infrared region.

Acknowledgements This work was funded by the EPSRC (EP/G04690 and EP/F028261). The research leading to these results has received funding from the European Community's Seventh Framework Programme (FP7/2007-2013) under grant agreement no PIEFGA-2008-221538. ISB was supported by a Research Fellowship from St John's College, Cambridge, UK. JH was supported by an Advanced Research Fellowship (EP/C012399/1) from the EPSRC. The authors thank Prof. R.L. Jones for his support and advice.

\section{References}

1. G. Berden, R. Engeln, Cavity Ring-Down Spectroscopy: Techniques and Applications (Wiley, New York, 2009)

2. J.J. Scherer, J.B. Paul, A. O'Keefe, R.J. Saykally, Chem. Rev. 97, 25 (1997)

3. M.D. Wheeler, S.M. Newman, A.J. Orr-Ewing, M.N.R. Ashfold, J. Chem. Soc. Faraday Trans. 94, 337 (1998)

4. G. Berden, R. Peeters, G. Meijer, Int. Rev. Phys. Chem. 19, 565 (2000)

5. S.S. Brown, Chem. Rev. 103, 5219 (2003)

6. J.M. Herbelin, J.A. McKay, M.A. Kwok, R.H. Ueunten, D.S. Urevig, D.J. Spencer, D.J. Benard, Appl. Opt. 19, 144 (1980)

7. R. Engeln, G. von Helden, G. Berden, G. Meijer, Chem. Phys. Lett. 262, 105 (1996) 
8. D. Romanini, A.A. Kachanov, F. Stoeckel, Chem. Phys. Lett. 270, 538 (1997)

9. M. Mazurenka, A.J. Orr-Ewing, R. Peverall, G.A.D. Ritchie, Ann. Rep. Prog. Chem., Sect. C 101, 100 (2005)

10. X. Mercier, P. Jamette, J.F. Pauwels, P. Desgroux, Chem. Phys. Lett. 305, 334 (1999)

11. S.M. Ball, R.L. Jones, Chem. Rev. 103, 5239 (2003)

12. R. Engeln, G. Berden, R. Peeters, G. Meijer, Rev. Sci. Instrum. 69, 3763 (1998)

13. A. O'Keefe, Chem. Phys. Lett. 293, 331 (1998)

14. S.M. Ball, J.M. Langridge, R.L. Jones, Chem. Phys. Lett. 398, 68 (2004)

15. J.M. Langridge, S.M. Ball, A.J.L. Shillings, R.L. Jones, Rev. Sci. Instrum. 79, 123110-1 (2008)

16. L.N. Seetohul, Z. Ali, M. Islam, Anal. Chem. 81, 4106 (2009)

17. S.E. Fiedler, A. Hese, A.A. Ruth, Chem. Phys. Lett. 371, 284 (2003)

18. J.C. Knight, P.St.J. Russell, Science 12, 276 (2002)

19. T. Laurila, R. Hernberg, Appl. Phys. Lett. 83, 845 (2003)

20. J. Hult, I.S. Burns, C.F. Kaminski, Appl. Opt. 44, 3675 (2005)

21. J. Hult, R.S. Watt, C.F. Kaminski, Opt. Express 15, 11385 (2007)

22. C.F. Kaminski, R.S. Watt, A.D. Elder, J.H. Frank, J. Hult, Appl. Phys. B 92, 367 (2008)

23. J. Langridge, T. Laurila, R.S. Watt, R.L. Jones, C.F. Kaminski, J. Hult, Opt. Express 16, 10188 (2008)

24. R.S. Watt, T. Laurila, C.F. Kaminski, J. Hult, Appl. Spectrosc. 63, 1389 (2009)

25. T. Gherman, D. Romanini, Opt. Express 10, 1033 (2002)

26. M.J. Thorpe, K.D. Moll, R.J. Jones, B. Safdi, J. Ye, Science 311, 1599 (2006)

27. P.S. Johnston, K.K. Lehmann, Opt. Express 16, 15013 (2008)

28. K. Stelmaszczyk, M. Fechner, P. Rohwetter, M. Queißer, A. Czyżewski, T. Stacewicz, L. Wöste, Appl. Phys. B-Lasers O 94, 193 (2009)
29. K. Stelmaszczyk, P. Rohwetter, M. Fechner, M. Queißer, A. Czyżewski, T. Stacewicz, L. Wöste, Opt. Express 17, 3673 (2009)

30. M. Schnippering, P.R. Unwin, J. Hult, T. Laurila, C.F. Kaminski, J.M. Langridge, R.L. Jones, M. Mazurenka, S.R. Mackenzie, Electrochem. Commun. 10, 1827 (2008)

31. L. van der Schneppen, G. Hancock, C. Kaminski, T. Laurila, S.R. Mackenzie, S.R.T. Neil, R. Peverall, G.A.D. Ritchie, P.R. Unwin, M. Schnippering, Analyst 135, 133 (2010)

32. K. Lehmann, P.S. Johnston, P. Rabinowitz, Appl. Opt. 48, 2966 (2009)

33. J.R. Lakowicz, Principles of Fluorescence Spectroscopy, 3rd edn. (Springer, Berlin, 2006)

34. L.S. Rothman, D. Jacquemart, A. Barbe, D.C. Benner, M. Birk, L.R. Brown, M.R. Carleer, C. Chackerian Jr., K. Chance, V. Dana, V.M. Devi, J.-M. Flaud, R.R. Gamache, A. Goldman, J.-M. Hartmann, K.W. Jucks, A.G. Maki, J.-Y. Mandin, S.T. Massie, J. Orphal, A. Perrin, C.P. Rinsland, M.A.H. Smith, J. Tennyson, R.N. Tolchenov, R.A. Toth, J. Vander Auwera, P. Varanasi, G. Wagner, J. Quant. Spectrosc. Radiat. Transf. 96, 139 (2005)

35. H.D. Babcock, L. Herzberg, Astrophys. J. 108, 167 (1948)

36. H. Naus, H. Ubachs, Appl. Optics 38, 3423 (1999)

37. P.L. Kebabian, W.A. Robinson, A. Freedman, Rev. Sci. Instrum. 78, 063102-1 (2007)

38. K. Bescherer, J.A. Barnes, S. Dias, G. Gagliardi, H.-P. Loock, N.R. Trefiak, H. Waechter, S. Yam, Appl. Phys. B-Lasers O 96, 193 (2009)

39. G. Meijer, M.G.H. Boogaarts, R.T. Jongma, D.H. Parker, A.M. Wodtke, Chem. Phys. Lett. 217, 112 (1994)

40. J.T. Hodges, J.P. Looney, R. van Zee, J. Chem. Phys. 105, 10278 (1996)

41. J. Martin, B.A. Paldus, P. Zalicki, E.H. Wahl, T.G. Owano, J.S. Harris, C.H. Kruger, R.N. Zare, Chem. Phys. Lett. 258, 63 (1996)

42. H. Huang, K. Lehmann, Opt. Express 15, 8745 (2007) 